\title{
Vital Signs Are Still Vital: Instability on Discharge and the Risk of Post-Discharge Adverse Outcomes
}

\author{
Oanh Kieu Nguyen, MD, MAS ${ }^{1,2}$, Anil N. Makam, MD, MAS ${ }^{1,2}$, Christopher Clark, MPA3, \\ Song Zhang, $P h D^{4}$, Bin Xie, $P h D^{5}$, Ferdinand Velasco, $M D^{6}$, Ruben Amarasingham, $M D, M B A^{7,2,5}$, \\ and Ethan A. Halm, MD, MPH ${ }^{1,2}$ \\ 'Division of General Internal Medicine, Department of Internal Medicine, UT Southwestern Medical Center, Dallas, TX, USA; ${ }^{2}$ Division of Outcomes \\ and Health Services Research, Department of Clinical Sciences, UT Southwestern Medical Center, Dallas, TX, USA; ${ }^{3}$ Office of Research \\ Administration, Parkland Health \& Hospital System, Dallas, TX, USA; ${ }^{4}$ Division of Biostatistics, Department of Clinical Sciences, UT Southwestern \\ Medical Center, Dallas, TX, USA; ${ }^{5} \mathrm{PCCl}$, Dallas, TX, USA; ${ }^{\circ}$ Texas Health Resources, Dallas, TX, USA.
}

BACKGROUND: Vital sign instability on discharge could be a clinically objective means of assessing readiness and safety for discharge; however, the association between vital sign instability on discharge and post-hospital outcomes is unclear.

OBJECTIVE: To assess the association between vital sign instability at hospital discharge and post-discharge adverse outcomes.

DESIGN: Multi-center observational cohort study using electronic health record data. Abnormalities in temperature, heart rate, blood pressure, respiratory rate, and oxygen saturation were assessed within 24 hours of discharge. We used logistic regression adjusted for predictors of 30-day death and readmission.

PARTICIPANTS: Adults ( $\geq 18$ years) with a hospitalization to any medicine service in 2009-2010 at six hospitals (safety-net, community, teaching, and non-teaching) in north Texas.

MAIN MEASURES: Death or non-elective readmission within 30 days after discharge.

KEY RESULTS: Of 32,835 individuals, 18.7 \% were discharged with one or more vital sign instabilities. Overall, $12.8 \%$ of individuals with no instabilities on discharge died or were readmitted, compared to $16.9 \%$ with one instability, $21.2 \%$ with two instabilities, and $26.0 \%$ with three or more instabilities $(p<0.001)$. The presence of any $(\geq 1)$ instability was associated with higher risk-adjusted odds of either death or readmission (AOR 1.36, $95 \% \mathrm{CI}$ 1.26-1.48), and was more strongly associated with death (AOR 2.31, $95 \%$ CI 1.91-2.79). Individuals with three or more instabilities had nearly fourfold increased odds of death (AOR 3.91, $95 \%$ CI 1.69-9.06) and increased odds of 30-day readmission (AOR 1.36, $95 \%$ 0.81-2.30) compared to individuals with no instabilities. Having two or more vital sign instabilities at discharge had a positive

$\overline{\text { Oanh Kieu Nguyen MD, MAS, and Anil N. Makam MD, MAS, contributed }}$ equally to this work.

Electronic supplementary material The online version of this article (doi:10.1007/s11606-016-3826-8) contains supplementary material, which is available to authorized users.

Received February 1, 2016

Revised June 2, 2016

Accepted July 14, 2016

Published online August 8, 2016 predictive value of $22 \%$ and positive likelihood ratio of 1.8 for 30-day death or readmission.

CONCLUSIONS: Vital sign instability on discharge is associated with increased risk-adjusted rates of 30-day mortality and readmission. These simple vital sign criteria could be used to assess safety for discharge, and to reduce 30-day mortality and readmissions.

$\mathrm{J}$ Gen Intern Med 32(1):42-8

DOI: $10.1007 / \mathrm{s} 11606-016-3826-8$

(c) Society of General Internal Medicine 2016

\section{INTRODUCTION}

Over the past 30 years, hospital length of stay has fallen dramatically, raising concern that many patients may be discharged before being stabilized. ${ }^{1,2}$ Premature discharge, as indicated by the presence of unresolved clinical instabilities at discharge, is associated with higher post-discharge mortality and readmission rates. The landmark RAND studies assessing the impact of the prospective payment system on Medicare beneficiaries hospitalized in the 1980s for heart failure, myocardial infarction, pneumonia, stroke, and hip fracture found that reduced length of stay was accompanied by a $43 \%$ relative increase in patients being discharged home in a clinically unstable state, and that those with at least one clinical instability had a $60 \%$ relative increase in the risk of death after discharge compared to those with no instabilities. ${ }^{3,4}$ Subsequent studies of patients hospitalized in the 1990s demonstrated that clinical instability on discharge remained common, and was associated with higher risk-adjusted odds of death or readmission among patients hospitalized for pneumonia or hip fracture. ${ }^{5,6}$

The Centers for Medicare \& Medicaid Services (CMS) public reporting of outcomes and the Hospital Readmission Reduction Program (HRRP) have both dramatically increased attention to reducing rates of both 30-day mortality and readmission. ${ }^{7-11}$ Despite intense efforts, attempts at prediction and prevention of these post-discharge adverse events have had mixed results. ${ }^{12-15}$ Assessing the stability of a patient's vital signs in the 24 hours prior to discharge is a simple, objective, and clinically sensible way of determining safety for discharge. 
Recognition and treatment of vital sign instabilities may also provide an easily actionable target to help providers and hospitals further reduce 30-day post-discharge adverse events. However, there have been no studies evaluating the association between vital sign instabilities at discharge and 30-day mortality and readmission in a general population of adults of all ages hospitalized for any medical condition, nor has this been examined in the current era of much shorter hospital stays and widespread interest in reducing readmissions.

To address this gap in the literature, we used a clinically granular electronic health record (EHR) dataset of diverse individuals hospitalized in a variety of settings to assess the rates and types of vital sign instability among hospitalized medical patients at discharge, as well as the association between vital sign instabilities at discharge and 30-day readmission and mortality even after adjusting for other prognostic factors. We hypothesized that the presence of vital sign instability would be associated with increased rates of 30-day mortality and readmission, and that an increasing number of instabilities would be associated with higher odds of an adverse post-discharge outcome.

\section{METHODS}

\section{Study Design, Population, and Data Sources}

We conducted an observational cohort study using EHR data collected from November 1, 2009, to October 30, 2010, from six hospitals in the Dallas-Fort Worth area, the fourth largest metroplex in the U.S. ${ }^{16}$ One site was a major safety-net hospital; the remaining five community hospitals were a mix of teaching and non-teaching facilities. All hospitals used the same EHR system (Epic Systems Corporation, Verona, WI). We included consecutive hospitalizations among adults 18 years of age or older who were discharged alive from a medicine inpatient service with any clinical diagnosis. For individuals who had multiple hospitalizations during this time period, we included only the first hospitalization. We excluded individuals who died in the hospital, were transferred to another acute care facility, or who left against medical advice. We also excluded those discharged to hospice, since these patients may have desired less aggressive care.

\section{Definition of Vital Sign Instability}

Vital sign instabilities were considered present at discharge if the most abnormal value for a particular vital sign met any of the following criteria within 24 hours of discharge: temperature $\geq 37.8^{\circ} \mathrm{C}$, heart rate $\geq 100$ beats per minute, respiratory rate $>24$ breaths per minute, systolic blood pressure $\leq 90 \mathrm{mmHg}$, or oxygen saturation $<90 \%$. These cutoffs for vital sign instability were based on clinical face validity and previously published studies. ${ }^{5,17}$ For the $<1 \%$ of individuals missing data within 24 hours of discharge, vital signs were classified as normal.

\section{Baseline Characteristics}

Utilization history, which included emergency department (ED) visits and hospitalizations in the 12 months preceding the index hospitalization, was ascertained from both the EHR and an all-payer regional hospitalization database which captures ED visits and hospitalizations from 75 acute care hospitals within 100 miles of Dallas. ${ }^{18}$ Hospital complications were defined using ICD-9-CM codes from the EHR for secondary diagnoses for the index hospitalization, and excluded conditions coded during any clinical encounter in the preceding year. Other baseline characteristics were obtained from the EHR.

\section{Outcomes}

Our primary outcome was a composite of death or readmission within 30 days of discharge. Readmissions were ascertained at the index hospital and at any of 75 acute care hospitals in the region, as described above. Individuals who had both outcomes (readmission and death) were only counted once in the primary outcome. Secondary outcomes were death and readmission within 30 days of discharge, considered as independent outcomes.

\section{Statistical Analysis}

To estimate the independent effect of vital sign instability on adverse outcomes, we used logistic regression, adjusting for factors identified in a previously validated multicondition EHR-based readmission and mortality risk model that included sociodemographic characteristics, utilization history, and clinical factors on admission and from the remainder of the hospitalization (comorbidities, laboratory abnormalities, hospital complications, etc.). ${ }^{19-29}$ To assess a dose-response relationship between an increasing number of instabilities and adverse outcomes, we used the Cochran-Armitage test for trend. We accounted for clustering of patients by hospital using generalized estimating equations. We assessed whether adverse outcomes among individuals with vital sign instabilities differed by disposition status (home, home health, versus post-acute care) using chi-square tests and logistic regression (with an interaction term for vital sign instability and disposition). We also assessed whether adverse outcomes among those with vital sign instabilities differed by whether or not the same instability was also present on admission, using chi-square tests. All analyses used Stata version 12.1 software (StataCorp LP, College Station, TX). The UT Southwestern Medical Center institutional review board approved this study and waived the requirement for informed consent.

\section{RESULTS}

We included a total of 32,835 unique individuals in the study cohort (Table 1; eFig. 1, Online Appendix). The mean age was 
$62 \pm 17$ years; over half were women, and individuals were diverse in race/ethnicity and primary insurance status. Most individuals had non-elective admissions, no previous ED visits or hospitalizations in the prior year, and had a Charlson comorbidity index of 0 . The overall median length of stay was 4 days (IQR 2-6 days).

\section{Types and Rates of Vital Sign Instabilities on Discharge}

Overall, 6127 individuals (18.7\%) had one or more instabilities on discharge. Elevated heart rate was the most common, affecting $10.4 \%$ of the study population (Table 2 ). The frequency of other specific instabilities and combinations of instabilities is shown in eTable 1 (Online Appendix). Combinations involving elevated temperature had the lowest unadjusted 30-day composite and mortality rates, but only a small number of individuals had a combination of instabilities, limiting further analysis. There were no significant differences in rates of instability on discharge among the six study sites (range $17.9 \%-20.3 \%$ ).

\section{Outcomes}

In the 30 days after discharge, 4484 (13.7\%) individuals had a readmission or death. The median time to the composite outcome was 12 days (IQR 5-20 days). A total of 4163 individuals were readmitted within 30 days (12.7\%), with a median time to readmission of 11 days (IQR 5-19 days). Only 535 individuals died within 30 days of discharge (1.6\%), with a median time to death of 16 days (IQR 9-24 days).

\section{Associations Between Vital Sign Instability on Discharge and Outcomes}

Figure 1 shows that the greater the number of instabilities on discharge, the greater risk of death and/or readmission. Overall, $12.8 \%$ of individuals with no instabilities on discharge died or were readmitted, compared to $16.9 \%$ with one instability, $21.2 \%$ with two instabilities, and $26.0 \%$ with three or more instabilities $(p<0.001)$.

Even after controlling for numerous other prognostic factors and potential confounders, including demographic, clinical, and utilization characteristics, we found a dose-dependent relationship between the number of vital sign instabilities and odds for 30-day post-discharge adverse events (Table 3; eTable 2, Online Appendix). The relationship was most striking for mortality, with the odds of death doubling, tripling, and quadrupling with each additional instability. Individuals with any instability on discharge had higher adjusted odds of the composite outcome (AOR 1.36, $95 \%$ CI 1.26-1.48), death (AOR 2.36, $95 \%$ CI 1.97-2.83), and readmission (AOR 1.36, $95 \% 1.26-1.47)$ compared to those discharged with stable vital signs (Table 3 ).

Table 1 Baseline Characteristics of Hospitalized Medical Patients

\begin{tabular}{|c|c|c|c|c|}
\hline & $\begin{array}{l}\text { Entire cohort } \\
(N=32,835)\end{array}$ & $\begin{array}{l}\text { No readmission } \\
\text { or death }(N=28,351)\end{array}$ & $\begin{array}{l}\text { Readmitted or died } \\
\text { within 30-days }(N=4484)\end{array}$ & $\overline{p \text { value }}$ \\
\hline Age in years, mean $\pm S D$ & $61.7 \pm 17.3$ & $61.2 \pm 17.3$ & $64.9 \pm 16.8$ & $\leq 0.001$ \\
\hline Female, $n(\%)$ & $17,589(53.6)$ & $15,196(53.6)$ & $2393(53.4)$ & 0.772 \\
\hline \multicolumn{4}{|l|}{ Race/ethnicity, $n(\%)$} & 0.020 \\
\hline White & $21,319(64.9)$ & $18,428(65.0)$ & $2891(64.5)$ & \\
\hline Black & $5944(18.1)$ & $5078(17.9)$ & $866(19.3)$ & \\
\hline Hispanic & $4422(13.5)$ & 3825 (13.5) & $597(13.3)$ & \\
\hline Other & $1150(3.5)$ & $1020(3.6)$ & $130(2.9)$ & \\
\hline \multicolumn{4}{|l|}{ Marital status, $n(\%)$} & $\leq 0.001$ \\
\hline Single & $8061(24.6)$ & $7007(24.7)$ & $1054(23.5)$ & \\
\hline Married & $13,402(40.8)$ & $11.764(41.5)$ & $1638(36.5)$ & \\
\hline Separated/divorced & 3465 (10.6) & $2929(10.3)$ & $536(11.9)$ & \\
\hline Widowed & $4447(13.5)$ & $3669(12.9)$ & $778(17.4)$ & \\
\hline Other & $3460(10.5)$ & $2982(10.5)$ & $478(10.7)$ & \\
\hline \multicolumn{4}{|l|}{ Primary insurance, $n(\%)$} & $\leq 0.001$ \\
\hline Private/commercial & $13,034(39.7)$ & $11,600(40.9)$ & $1434(32.0)$ & \\
\hline Medicare & $12,995(39.6)$ & $10,863(38.2)$ & $2132(47.5)$ & \\
\hline Medicaid & $2192(6.7)$ & $1719(6.1)$ & $473(10.6)$ & \\
\hline Charity, self-pay, or other & $4614(14.0)$ & 4169 (14.7) & $445(9.9)$ & \\
\hline Non-elective admission, $n(\%) *$ & $27,726(84.4)$ & $23,730(83.7)$ & $3996(89.1)$ & $\leq 0.001$ \\
\hline Emergency department visits in prior 12 months, $n(\%)$ & $9249(28.2)$ & 7619 (26.9) & $1630(36.3)$ & $\leq 0.001$ \\
\hline Hospitalizations in prior 12 months, $n(\%)$ & $10,163(30.9)$ & $8098(28.6)$ & $2065(46.1)$ & $\leq 0.001$ \\
\hline \multicolumn{4}{|l|}{ Charlson comorbidity index, $n(\%)^{\dagger}$} & $\leq 0.001$ \\
\hline 0 & $24,249(73.9)$ & $21,655(76.4)$ & $2594(57.9)$ & \\
\hline 1 & $1916(5.8)$ & $1615(5.7)$ & $301(6.7)$ & \\
\hline $2+$ & $6670(20.3)$ & $5081(17.9)$ & $1589(35.4)$ & \\
\hline \multicolumn{5}{|l|}{ Hospital complications, $n(\%)$} \\
\hline Clostridium difficile infection & $122(0.4)$ & $83(0.3)$ & $39(0.9)$ & $<0.001$ \\
\hline Pressure ulcer & $365(1.1)$ & $262(0.9)$ & $103(2.3)$ & $\leq 0.001$ \\
\hline Venous thromboembolism & $298(0.9)$ & $228(0.8)$ & $70(1.6)$ & $\leq 0.001$ \\
\hline Length of stay, median (IQR) & $4(2-6)$ & $4(2-6)$ & $5(3-8)$ & $\leq 0.001$ \\
\hline
\end{tabular}

IQR interquartile range

*Non-elective defined as hospitalization categorized as medical emergency, urgent, or trauma

${ }^{+}$Charlson comorbidity index was calculated using the Deyo modification 


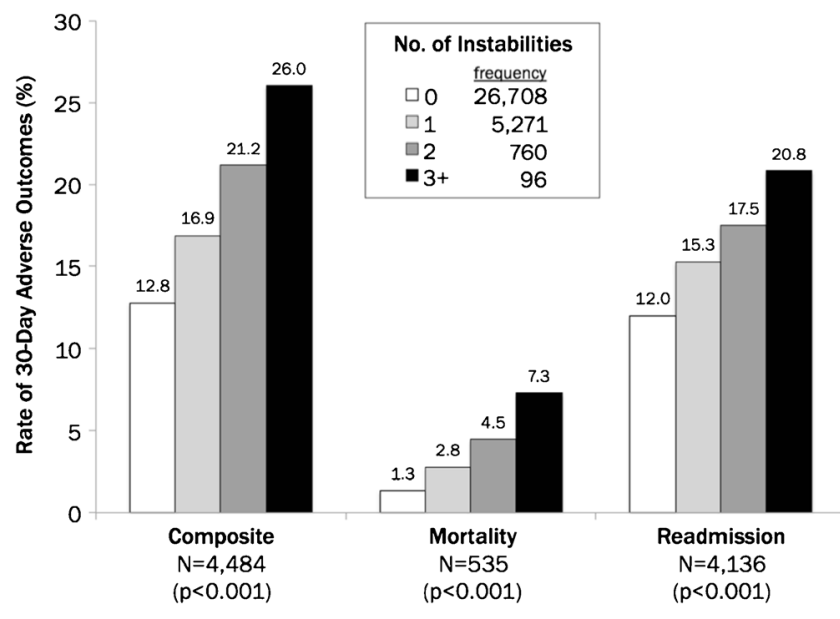

Figure 1 Rates of 30-day mortality and readmission stratified by number of instabilities at discharge.

\section{Associations Between Instability on Discharge and Disposition Status}

The greater the number of vital sign instabilities an individual had on hospital discharge, the more likely they were to be discharged to a post-acute care facility (which included nursing homes, skilled nursing facilities, and long-term acute care facilities; eFig. 2, Online Appendix). Only $18.1 \%$ of individuals with no instabilities were discharged to post-acute care, compared to $21.5 \%$ of those with one instability, $26.7 \%$ of those with two instabilities, and $42.7 \%$ of those with three or more instabilities ( $p$ value $<0.001$ for trend). Although instability on discharge remained a significant predictor of death and readmission irrespective of disposition status, rates of post-discharge adverse events were uniformly much higher among those discharged to a post-acute care facility compared to those discharged home ( $p$ value $<0.001$ for interaction). For those with two instabilities, $12.3 \%$ of individuals discharged to a post-acute care facility died and $18.7 \%$ were readmitted within 30-days, compared to 30-day mortality and readmission rates of $1.6 \%$ and $17.1 \%$, respectively, among those discharged home ( $p<0.001$ for both comparisons).

\section{Onset of Vital Sign Instabilities}

The proportion of patients with the same instability present on admission ranged from $38 \%$ for fever to $76 \%$ for tachycardia (eTable 3, Online Appendix). However, rates of postdischarge adverse outcomes did not differ by whether the instability was also present on admission.

\section{Assessment of Instability on Discharge as a Diagnostic Test for Adverse Events}

From a clinical perspective, individual physicians or hospitals may want to use a specific definition of instability to help gauge safety and appropriateness of discharge. Thus, vital sign instability criteria may be considered a type of diagnostic test for future adverse events. The test characteristics, including sensitivity, specificity, positive and negative predictive values, and positive and negative likelihood ratios, for various cutoffs used to define instability are displayed in Table 4 . A greater number of vital sign instabilities is more helpful in predicting death within 30 days ( $\mathrm{LR}^{+} 3.03,95 \%$ CI $2.25-4.11$ for $\geq 2$ instabilities) than readmission $\left(\mathrm{LR}^{+} 1.50,95 \% \mathrm{CI} 1.26-1.78\right.$ for $\geq 2$ instabilities). However, the absence of vital sign instability does not necessarily rule out these outcomes (i.e., $\mathrm{LR}^{-}$range of 0.8-1.0).

\section{DISCUSSION}

In this multi-center observational cohort study, with a diverse mix of community, teaching, and public safety-net hospitals caring for patients with substantial diversity in age, race/ethnicity, insurance status and clinical conditions, nearly one in five hospitalized patients on a medicine service were discharged from the hospital with one or more vital sign instabilities. Leaving the hospital before vital signs were clinically stable was associated with important clinical consequences - the greater the number of vital sign instabilities, the greater the risk of death or readmission within 30 days. Individuals with any vital sign instability had nearly $40 \%$ increased odds of death or readmission within 30 days of discharge, even after adjusting for other important prognostic factors and potential confounders. Among individuals with two or more vital signs instabilities, the odds of death

Table 2 Overall Frequency of Vital Sign Instabilities on Discharge

\begin{tabular}{|c|c|c|c|c|}
\hline & \multicolumn{3}{|l|}{ n (\%) } & \multirow[t]{2}{*}{$p$ value } \\
\hline & $\begin{array}{l}\text { Entire cohort } \\
(\mathbf{N}=\mathbf{3 2 , 8 3 5 )}\end{array}$ & $\begin{array}{l}\text { No readmission } \\
\text { or death }(N=28,351)\end{array}$ & $\begin{array}{l}\text { Readmitted or died } \\
\text { within } 30 \text { days }(N=4484)\end{array}$ & \\
\hline Any instabilities present & $6,127(18.7)$ & $5051(17.8)$ & $1076(24.0)$ & $<0.001$ \\
\hline 1 & $5,271(16.1)$ & $4381(15.5)$ & $890(19.9)$ & $<0.001$ \\
\hline 2 & $760(2.3)$ & $599(2.1)$ & $161(3.6)$ & \\
\hline $3+$ & $96(0.4)$ & $71(0.3)$ & $25(0.6)$ & \\
\hline \multicolumn{5}{|l|}{ Instability at discharge } \\
\hline Temperature $\geq 37.8^{\circ} \mathrm{C}$ & $504(1.5)$ & $428(1.5)$ & $76(1.7)$ & 0.348 \\
\hline Respiratory rate $>24$ breaths/min & $825(2.5)$ & $680(2.4)$ & $145(3.2)$ & 0.001 \\
\hline Heart rate $>100$ beats $/ \mathrm{min}$ & $3,410(10.4)$ & $2785(9.8)$ & $625(13.9)$ & $<0.001$ \\
\hline Systolic blood pressure $\leq 90 \mathrm{mmHg}$ & $1,299(4.0)$ & $1056(3.7)$ & $243(5.4)$ & $<0.001$ \\
\hline Oxygen saturation $<90 \%$ & $1,050(3.2)$ & $850(3.0)$ & $200(4.5)$ & $<0.001$ \\
\hline
\end{tabular}


Table 3 Associations Between Vital Sign Instability on Discharge and 30-Day Mortality and Readmission*

\begin{tabular}{|c|c|c|c|c|c|c|}
\hline & \multicolumn{5}{|c|}{ Number of instabilities on discharge } & \multirow[t]{2}{*}{$p$ value $^{\dagger}$} \\
\hline & Any & $\mathbf{0}$ & 1 & 2 & $3+$ & \\
\hline \multicolumn{7}{|l|}{ Composite } \\
\hline Unadjusted & $1.5(1.4-1.6)$ & [Reference] & $1.4(1.3-1.5)$ & $1.8(1.5-2.2)$ & $2.4(1.5-3.8)$ & \multirow[t]{2}{*}{$<0.001$} \\
\hline Adjusted ${ }^{\ddagger}$ & $1.4(1.3-1.5)$ & [Reference] & $1.3(1.2-1.4)$ & $1.7(1.4-2.0)$ & $1.7(1.0-2.8)$ & \\
\hline \multicolumn{7}{|l|}{ Mortality } \\
\hline Unadjusted & $2.4(2.0-2.8)$ & [Reference] & $2.1(1.8-2.6)$ & $3.5(2.5-5.1)$ & $5.9(2.7-12.9)$ & \multirow[t]{2}{*}{$<0.001$} \\
\hline Adjusted ${ }^{\ddagger}$ & $2.3(1.9--2.8)$ & [Reference] & $2.1(1.7-2.6)$ & $3.3(2.3-4.9)$ & $3.9(1.7-9.1)$ & \\
\hline \multicolumn{7}{|l|}{ Readmission } \\
\hline Unadjusted & $1.4(1.3-1.5)$ & [Reference] & $1.3(1.2-1.4)$ & $1.6(1.3-1.9)$ & $1.9(1.2-3.2)$ & \multirow{2}{*}{$<0.001$} \\
\hline Adjusted & $1.3(1.2-1.4)$ & [Reference] & $1.2(1.1-1.4)$ & $1.4(1.2-1.7)$ & $1.4(0.8-2.3)$ & \\
\hline
\end{tabular}

*Data are odds ratio (95\% confidence interval) unless otherwise specified

${ }^{*}$ According to Cochran-Armitage test for trend comparing the outcomes of patients with 0, 1, 2, and 3 or more instabilities on discharge

* Adjusted for demographic characteristics (age, Medicaid, widow), utilization history (ED visits and hospitalizations in previous 12 months), clinical factors on admission (factors were ascertained within 24 hours of admission and included non-elective admission, Charlson comorbidity index, laboratory abnormalities [creatine phosphokinase $<60 \mathrm{mcg} / \mathrm{L}$, mean corpuscular volume $>100 \mathrm{fL} /$ red cell, platelets $<90 \times 10^{3} / \mu \mathrm{L}$, and prothrombin time $>35$ seconds]), and clinical factors from the rest of the hospitalization (length of stay; complications [Clostridium difficile infection, pressure ulcer, and venous thromboembolism]; and lab abnormalities on discharge [blood urea nitrogen $>20 \mathrm{mg} / \mathrm{dL}$, serum sodium $<135 \mathrm{mEq} / \mathrm{L}$, and hematocrit $\leq 27 \%$ ]; values from within 24 hours of discharge). We used cutoffs for lab values from prior literature; some of these cutoffs were originally defined through recursive partitioning ${ }^{19-29}$

increased more than threefold. Lastly, patients were more likely to be sent to post-acute care facilities with an increasing number of vital sign instabilities at discharge, and experienced much higher 30-day mortality and readmission rates compared to unstable patients discharged home. In other words, discharge to a post-acute care institution did not diminish the association between vital sign instabilities and adverse events.

To our knowledge, this is the first study on the effect of vital sign instabilities on post-discharge adverse outcomes in a general population of adults hospitalized for any medical condition, especially in the era of much shorter hospital stays and focus on reducing readmissions. Our findings confirm and extend the landmark studies by RAND and Halm et al., which assessed the effect of more broadly defined clinical instabilities on discharge in groups with selected conditions in the 1980s and 1990s, respectively. ${ }^{4,5}$ Overall, these studies found that $15-19 \%$ of hospitalized patients had at least one instability on discharge, and that having any instability was associated with significantly increased risk of 30-day mortality and readmission. In contrast to the prior RAND and Halm studies, we deliberately used a more parsimonious definition of clinical instability based only on vital signs, because these factors are routinely measured in clinical practice, are routinely collected in the EHR, are easy to query and extract electronically, and are simple, common and objective data routinely used by many providers to assess clinical status and readiness for discharge. Additionally, unlike many other factors identified by prediction models as being associated with increased risk for post-discharge adverse outcomes (e.g., prior utilization), vital sign instabilities provide a direct mechanistic link between severity and trajectory of illness and subsequent 30day adverse outcomes. We also included a broader population of adults of all ages hospitalized with any medical condition, so our findings are more generalizable. Nearly 30 years after the initial RAND study, we found that vital sign instabilities on discharge remain a prevalent and robust predictor of risk-adjusted 30-day adverse post-hospital events. Simply put, vital signs are still vital.

Our findings suggest that the presence of one or more vital sign instabilities should signal that these patients should be discharged with caution, given that the adjusted odds of death after discharge more than doubled when a single vital sign instability was present within 24 hours of discharge. However, given the modest absolute risk and the substantial proportion of individuals with one or more instabilities (18.7\% of our population), clinicians should carefully weigh the risks versus benefits of extending hospitalization in this group and tailor discharge practices accordingly. The presence of two or more vital sign instabilities should strongly be considered as objective criteria to judge readiness for discharge among all medical inpatients, given that the adjusted odds of death more than tripled among this small (2.6\% of our population) but high-

Table 4 Diagnostic Test Characteristics of Vital Sign Instabilities on Discharge to Detect Adverse 30-Day Post-Discharge Outcomes

\begin{tabular}{|c|c|c|c|c|c|c|c|}
\hline$\overline{\text { Outcome }}$ & No. of instabilities & Sensitivity, \% & Specificity, \% & PPV, \% & NPV, \% & $\overline{\mathbf{L R}^{+}}$ & $\overline{\mathbf{L R}^{-}}$ \\
\hline \multirow[t]{3}{*}{ Composite } & $\geq 1$ & 24.0 & 82.2 & 17.6 & 87.2 & 1.3 & 0.9 \\
\hline & $\geq 2$ & 4.1 & 97.6 & 21.7 & 86.6 & 1.8 & 1.0 \\
\hline & $\geq 3$ & 0.6 & 99.8 & 26.0 & 86.4 & 2.2 & 1.0 \\
\hline \multirow[t]{3}{*}{ Mortality } & $\geq 1$ & 34.8 & 81.6 & 3.0 & 98.7 & 1.9 & 0.8 \\
\hline & $\geq 2$ & 7.7 & 97.5 & 4.8 & 98.5 & 3.0 & 0.9 \\
\hline & $\geq 3$ & 1.3 & 99.7 & 7.3 & 98.4 & 4.8 & 1.0 \\
\hline \multirow[t]{3}{*}{ Readmission } & $\geq 1$ & 23.0 & 82.0 & 15.6 & 88.0 & 1.3 & 0.9 \\
\hline & $\geq 2$ & 3.7 & 97.5 & 17.9 & 87.5 & 1.5 & 1.0 \\
\hline & $\geq 3$ & 0.5 & 99.7 & 20.8 & 87.3 & 1.8 & 1.0 \\
\hline
\end{tabular}

PPV positive predictive value, NPV negative predictive value, $L R^{+}$positive likelihood ratio, $L R^{-}$negative likelihood ratio 
risk group of individuals. However, one limitation is that we do not know whether extending the hospitalization for individuals with vital sign instabilities would result in clinical stabilization. ${ }^{30,31}$ Further research is needed to establish whether an intervention strategy extending the acute care hospital stay for patients with vital sign instabilities would result in stabilization and fewer post-discharge adverse events among a broad population of hospitalized patients. This will be critical to the ongoing development of objective, evidence-based discharge criteria to optimize post-discharge patient safety.

Our finding that patients with a greater number of vital sign instabilities were more likely to be discharged to a post-acute facility suggests that post-acute care may be perceived as an acceptable alternate to longer hospitalizations for these sicker individuals. Several studies of the impact of the Medicare Inpatient Prospective Payment System (PPS), which created strong financial incentives to shorten hospital stays, found increasing rates of discharge to post-acute care after adoption of PPS. ${ }^{32,33}$ This practice pattern may have been further reinforced by implementation of Medicare's Hospital Readmission Reduction Program in 2012, which financially penalizes hospitals for high risk-adjusted readmissions, but specifically excludes readmissions from post-acute care facilities. ${ }^{3,34-36}$ However, the higher rates of 30-day post-discharge adverse events among those discharged to post-acute care facilities with vital sign instabilities suggests that even with the resources and closer monitoring typically offered in these settings, post-acute care facilities may not be equipped to stabilize and treat patients with vital sign instabilities. Furthermore, an alternate disposition - either prolonged hospitalization or end-of-life care planning, given markedly higher mortality rates - may have been more appropriate for a significant subset of these individuals. A better understanding of the role of post-acute care for individuals with vital sign instabilities at discharge is needed, and will become a more pressing issue with anticipated payment reform measures to encourage greater integration and accountability for outcomes across the entire spectrum of care. . $3,37-39^{-3}$

Our study had several strengths. First, our cohort was diverse with respect to hospital type and patient population. Second, we were able to use a validated risk-adjustment model based on detailed, clinically granular information in the EHR to adjust for numerous other poor prognostic factors, including sociodemographic characteristics, severity on admission, comorbid illness burden, utilization, hospital complications, and physiological trajectory. Third, we included all medical inpatient admissions, rather than focusing on selected diagnoses; thus, our findings may be more generalizable than those of previous studies. ${ }^{4,5}$ Fourth, we were also able to ascertain readmissions within a large geographic region rather than only within our six-hospital cohort.

Our study had certain limitations. Because this was an observational study, we cannot unambiguously infer causality. Findings may be different in other regions with different hospital discharge practices and post-acute care referral patterns. There may have been some patients who were discharged home prior to attaining stability because the patient and/or physician desired intentionally less aggressive care; however, this was one of the reasons we excluded individuals discharged to hospice from our analysis. Because we did not have data on all vital signs in the 24 hours prior to discharge (only the highest and lowest), it is possible that some patients may have had one or more sets of normal vital signs prior to discharge. Nonetheless, our findings suggest that providers should pay close attention to patients' vital signs in the 24 hours prior to contemplating discharge, and not just the very last set, which may be subject to measurement bias favoring discharge.

\section{CONCLUSIONS}

Vital sign instabilities in the 24 hours prior to discharge are common, and are associated with increased riskadjusted rates of 30-day adverse post-discharge events, particularly death. Discharge guidelines should include vital sign criteria for judging stability on discharge in order to improve disposition planning and post-discharge patient safety. At a minimum, patients with one instability on discharge should be discharged with caution. Close outpatient follow-up and appropriate patient education about warning signs and symptoms that merit urgent medical attention may also be warranted. Individuals with two or more instabilities should likely remain in the hospital for continued treatment and observation in the absence of extenuating circumstances. Though post-acute care facilities are increasingly providing care for patients discharged with vital sign instabilities, they may not be optimally equipped to care for these individuals. Further attention is needed toward developing evidence-based discharge criteria and interventions to optimize post-discharge patient safety.

Acknowledgments: This study was presented at the Society of Hospital Medicine 2015 Annual Meeting in National Harbor, MD, and the Society of General Internal Medicine 2015 Annual Meeting in Toronto, Canada. Drs. Nguyen and Makam and Mr. Clark had full access to all the data in the study and take responsibility for the integrity of the data and the accuracy of the data analysis. This work was supported by grant funding from the Agency for Healthcare Research and Quality UT Southwestern Center for Patient-Centered Outcomes Research (1R24HSO22418-01) and the Commonwealth Foundation (\#20100323). Drs. Nguyen and Makam received funding from the UT Southwestern KL2 Scholars Program (NIH/NCATS KL2 TR001103). Dr. Halm was also supported in part by NIH/NCATS U54 RFA-TR-12-006. The study sponsors had no role in the design or conduct of the study; collection, management, analysis, or interpretation of the data; or preparation, review, or approval of the manuscript.

Corresponding Author: Oanh Kieu Nguyen, MD, MAS; Division of General Internal Medicine, Department of Internal Medicine, UT Southwestern Medical Center, Dallas, TX, USA (e-mail: OanhK.Nguyen@UTSouthwestern.edu).

\section{Compliance with Ethical Standards:}

Conflict of Interest: The authors declare that they do not have a conflict of interest. 


\section{REFERENCES}

1. Buie VC, Owings MF, DeFrances CJ, Golosinskiy A. National hospital discharge survey: 2006 summary. Vital Health Stat. 2006;2010:13(168).

2. Weiss AJTHA, Barrett MLMLB, Inc.), Steiner CAA. Trends and Projects in Inpatient Hospital Costs and Utilization, 2003-2013. HCUP Statistical Brief \# 175. Rockville, MD.July 2014.

3. Kahn KL, Keeler EB, Sherwood MJ, et al. Comparing outcomes of care before and after implementation of the DRG-based prospective payment system. JAMA. 1990;264(15): 1984-1988.

4. Kosecoff J, Kahn KL, Rogers WH, et al. Prospective payment system and impairment at discharge. The 'quicker-and-sicker' story revisited. JAMA. 1990;264(15): 1980-1983.

5. Halm EA, Fine MJ, Kapoor WN, Singer DE, Marrie TJ, Siu AL. Instability on hospital discharge and the risk of adverse outcomes in patients with pneumonia. Arch Intern Med. 2002;162(11):1278-1284.

6. Halm EA, Magaziner J, Hannan EL, et al. Frequency and impact of active clinical issues and new impairments on hospital discharge in patients with hip fracture. Arch Intern Med. 2003;163(1):108-113.

7. Krumholz HM, Normand SL. Public reporting of 30-day mortality for patients hospitalized with acute myocardial infarction and heart failure. Circulation. 2008;118(13): 1394-1397.

8. U.S. Department of Health and Human Services. Hospital Compare. http://www.hospitalcompare.hhs.gov. Accessed 20 June 2016.

9. Fung CH, Lim YW, Mattke S, Damberg C, Shekelle PG. Systematic review: the evidence that publishing patient care performance data improves quality of care. Ann Intern Med. 2008;148(2):111-123.

10. Joynt KE, Jha AK. Thirty-day readmissions-truth and consequences. N Engl J Med. 2012;366(15):1366-1369.

11. Kangovi S, Grande D. Hospital readmissions-not just a measure of quality. JAMA. 2011;306(16):1796-1797.

12. Kansagara D, Englander $\mathbf{H}$, Salanitro A, et al. Risk prediction models for hospital readmission: a systematic review. JAMA. 2011;306(15): 1688-1698.

13. McCrum ML, Joynt KE, Orav EJ, Gawande AA, Jha AK. Mortality for publicly reported conditions and overall hospital mortality rates. JAMA Intern Med. 2013;173(14):1351-1357.

14. Hansen LO, Young RS, Hinami K, Leung A, Williams MV. Interventions to reduce 30-day rehospitalization: a systematic review. Ann Intern Med. 2011;155(8):520-528.

15. Bradley EH, Curry LA, Spatz ES, et al. Hospital strategies for reducing risk-standardized mortality rates in acute myocardial infarction. Ann Intern Med. 2012;156(9):618-626

16. United States Census Bureau. Annual Estimates of the Population of Metropolitan and Micropolitan Statistical Areas: April 1, 2010 to July 1, 2014. March 2015.

17. Akram AR, Chalmers JD, Taylor JK, Rutherford J, Singanayagam A, Hill AT. An evaluation of clinical stability criteria to predict hospital course in community-acquired pneumonia. Clin Microbiol Infect. 2013;19(12):1174-1180.

18. Dallas Fort Worth Hospital Council. Data Services. https://dfwhcfoundation.org/data. Accessed 20 June 2016.

19. Amarasingham R, Velasco F, Xie B, et al. Electronic medical recordbased multicondition models to predict the risk of 30 day readmission or death among adult medicine patients: validation and comparison to existing models. BMC Med Inform Decis Making. 2015;15(1):39.

20. Nguyen OK, Makam AN, Clark C, et al. Predicting all-cause 30-day hospital readmissions using electronic health record data over the course of hospitalization: model derivation, validation, and comparison. J Hosp Med. 2016;11(7):473-80.

21. Encinosa WE, Hellinger FJ. The impact of medical errors on ninety-day costs and outcomes: an examination of surgical patients. Health Serv Res. 2008;43(6):2067-2085.
22. Friedman B, Encinosa W, Jiang HJ, Mutter R. Do patient safety events increase readmissions? Med Care. 2009;47(5):583-590.

23. Miller RD, Eng T, Kandilov A, Cromwell J, McCall NT. Readmissions Due to Hospital-Acquired Conditions (HACs): Multivariate Modeling and Timing of Clinical Presentation Analyses. 2012.

24. Khong CJ, Baggs J, Kleinbaum D, Cochran R, Jernigan JA. The likelihood of hospital readmission among patients with hospital-onset central line-associated bloodstream infections. Infect Control Hosp Epidemiol. 2015:1-7.

25. Chopra T, Neelakanta A, Dombecki C, et al. Burden of Clostridium difficile infection on hospital readmissions and its potential impact under the Hospital Readmission Reduction Program. Am J Infect Control. 2015;43(4):314-317.

26. Bradley EH, Sipsma H, Horwitz LI, et al. Hospital strategy uptake and reductions in unplanned readmission rates for patients with heart failure: a prospective study. J Gen Intern Med. 2015;30(5):605611 .

27. Coca SG, Yusuf B, Shlipak MG, Garg AX, Parikh CR. Long-term risk of mortality and other adverse outcomes after acute kidney injury: a systematic review and meta-analysis. Am J Kidney Dis. 2009;53(6):961973.

28. Wald R, Jaber BL, Price LL, Upadhyay A, Madias NE. Impact of hospitalassociated hyponatremia on selected outcomes. Arch Intern Med. 2010;170(3):294-302.

29. Koch CG, Li L, Sun Z, et al. Hospital-acquired anemia: prevalence, outcomes, and healthcare implications. J Hosp Med. 2013;8(9):506-512.

30. Halm EA, Fine MJ, Marrie TJ, et al. Time to clinical stability in patients hospitalized with community-acquired pneumonia: implications for practice guidelines. JAMA. 1998;279(18): 1452-1457.

31. Takada K, Matsumoto S, Kojima E, et al. Predictors and impact of time to clinical stability in community-acquired pneumococcal pneumonia. Respir Med. 2014;108(5):806-812.

32. Kenney G, Holahan J. Nursing home transfers and mean length of stay in the prospective payment era. Med Care. 1991;29(7):589-609.

33. Burke RE, Juarez-Colunga E, Levy C, Prochazka AV, Coleman EA, Ginde AA. Patient and Hospitalization Characteristics Associated With Increased Postacute Care Facility Discharges From US Hospitals. Med Care. 2015;53(6):492-500.

34. Centers for Medicare and Medicaid Services, Department of Health and Human Services. Medicare program; hospital inpatient prospective payment systems for acute care hospitals and the long-term care hospital prospective payment system and fiscal year 2015 rates; quality reporting requirements for specific providers; reasonable compensation equivalents for physician services in excluded hospitals and certain teaching hospitals; provider administrative appeals and judicial review; enforcement provisions for organ transplant centers; and electronic health record (EHR) incentive program. Final rule. Fed Regist. 2014;79(163):49853-50536.

35. Centers for Medicare \& Medicaid Services. Readmissions Reduction Program. http://www.cms.gov/Medicare/Medicare-Fee-for-Service-Payment/AcuteInpatientPPS/Readmissions-Reduction-Program.html. Accessed 20 June 2016.

36. McIlvennan CK, Eapen ZJ, Allen LA. Hospital readmissions reduction program. Circulation. 2015;131(20): 1796-1803.

37. Newhouse JP, Garber AM. Geographic variation in health care spending in the United States: insights from an Institute of Medicine report. JAMA. 2013;310(12):1227-1228.

38. Chandra A, Dalton MA, Holmes J. Large increases in spending on postacute care in Medicare point to the potential for cost savings in these settings. Health Aff. 2013;32(5):864-872.

39. Mechanic R. Post-acute care-the next frontier for controlling Medicare spending. N Engl J Med. 2014;370(8):692-694. 